\title{
Planning of Truck Platoons: a Literature Review and Directions for Future Research
}

\author{
Anirudh Kishore Bhoopalam ${ }^{\mathrm{a}, *}$, Niels Agatz ${ }^{\mathrm{a}}$, Rob Zuidwijk ${ }^{\mathrm{a}}$ \\ ${ }^{a}$ Rotterdam School of Management, Erasmus University \\ Department of Technology $\&$ Operations Management \\ Mandeville (T) Building, Burgemeester Oudlaan 50 \\ 3062PA Rotterdam, The Netherlands
}

\begin{abstract}
A truck platoon is a set of virtually linked trucks that drive closely behind one another using automated driving technology. Benefits of truck platooning include cost savings, reduced emissions, and more efficient use of road capacity. To fully reap these benefits in the initial phases of technology deployment, careful planning of platoons based on trucks' itineraries and time schedules is required. This paper provides a framework to classify various new transportation planning problems that arise in truck platooning, surveys relevant operations research models for these problems in the literature and identifies directions for future research.

Keywords: Truck platooning, Optimization, Transportation, Automated driving, Review paper
\end{abstract}

\section{Introduction}

Novel semi-automated driving technologies, collectively referred to as Cooperative Adaptive Cruise Control (CACC), enable trucks to drive very close together as a platoon. Trucks in a platoon are virtually linked and communicate with each other through wireless communication technology. The leading truck is manually driven at the first position of the platoon and automatically followed by one or more following trucks. This means that the following trucks automatically brake, steer and (de)accelerate based on the actions of the

\footnotetext{
${ }^{*}$ Corresponding author
}

Email addresses: kishorebhoopalam@rsm.nl (Anirudh Kishore Bhoopalam), nagatz@rsm.nl (Niels Agatz), rzuidwijk@rsm.nl (Rob Zuidwijk) 
leading truck.

Truck platooning has been the subject of heightened interest recently because of the when the deployment of platooning technology is not widespread or on routes with little 
freight traffic, careful centralized planning is required to create platoons (Janssen et al., 2015). A so-called platooning service provider (Roland Berger, 2016; Janssen et al., 2015) could organize the planning and control of platoons between different fleets. Platoons could be scheduled in advance or planned in real-time during execution.

To establish a platoon, the departure times, travel speeds and the routes of the trucks in the platoon must be synchronized. A truck may, for instance, adjust its route and possibly even make a small detour to join a platoon. Figure 1 depicts an example of a platoon between two trucks in which one of the trucks makes a detour to form the platoon.

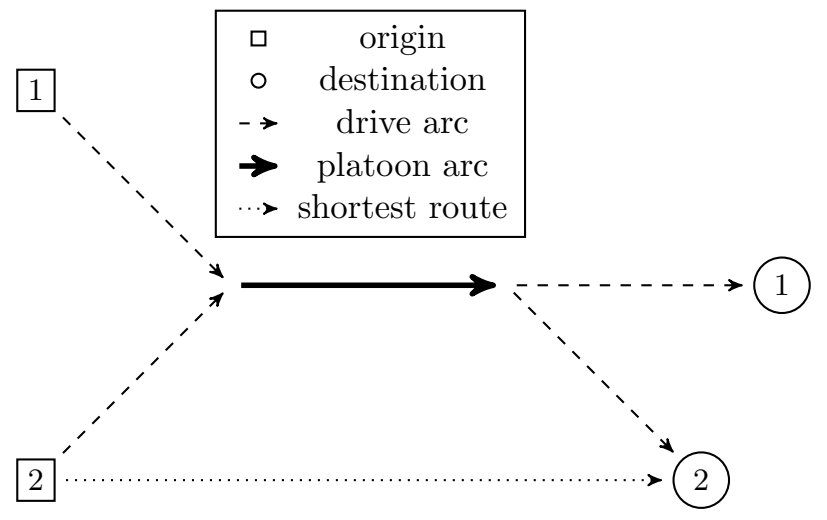

Figure 1: A two-truck platoon

While creating platoons, complex planning problems may arise, especially with multiple platoons per trip while considering detours. To fully reap the benefits of truck platooning, now and in the future, sophisticated decision support models and tools are required. Such models are not only useful to support platooning operations but can also help quantify the potential benefits of different types of platooning. While there has been much attention for the technological issues (see Berenghem et al. (2012) for an overview of recent projects), safe manoeuvring of platoons (see Kavathekar and Chen (2011) for an overview), human factors (for example, Heikoop et al. (2017); Hjamdähl et al. (2017); Yamabe et al. (2012); Larburu et al. (2010)), we are not aware of a paper that systematically reviews the challenges of platooning from a planning and transportation optimization perspective.

This paper aims to fill the above mentioned gap by classifying the different planning problems that arise in truck platooning, reviewing the emerging literature related to this planning and identifying directions for future research. More specifically, the goal of this 
paper is fourfold: (1) provide a systematic overview of different forms of platooning; (2) (3) provide an overview of relevant operations research models and approaches for these problems in the literature and; (4) identify gaps and areas for future research.

This paper focuses on truck platooning but similar issues may arise in the planning of platoons of regular cars and other vehicles not only on the road but also on water (see Lauf 
routes between the origin and the destination. The trip announcement also specifies the characteristics of the truck and its load and could contain preferences for the position within the platoon.

A platoon plan specifies (1) which trucks platoon together, (2) where and when the trucks form a platoon, (3) the route the platoon will take and (4) in what sequence the trucks drive within the platoon. Based on these platoon plans, the different trucks then typically form platoons en-route. For more information about the en-route formation of a platoon while interacting with surrounding traffic, see e.g. Segata et al. (2014); Berenghem et al. (2012). Trucks can wait for one another to form a platoon or can catch up by adjusting their driving speeds. To catch up, the truck that is behind can speed up or the truck that is in front can slow down. However, due to speed limits, forming platoons in this way is likely to require too much time in most practical settings.

Depending on when the trip announcements become available, we can distinguish the following three situations.

Scheduled platoon planning All trips are announced before the start of the operations. Therefore, all platoon plans can be created in advance. This is often referred to as off-line or static planning.

Real-time platooning Truck operators announce their trips closely before departure or even when the trucks are en-route. Therefore, trip announcements arrive during the execution of the trips. This is often referred to as online or dynamic planning.

Opportunistic platooning Trucks that are in close proximity of each other form platoons dynamically on the road without any prior planning. This type of platooning is also referred to as spontaneous, ad-hoc or on the fly platooning (Janssen et al., 2015; Liang et al., 2014).

When the deployment of platooning technology is not widespread, opportunistic platooning will not be very successful in creating platoons and therefore some form of planning is required. A simulation study by Liang et al. (2014) shows that there are substantial benefits associated with the careful planning of platoons. In the subsequent sections of the paper, we 
first discuss various aspects of scheduled platoon planning and then highlight the additional challenges in real-time platoon planning.

\subsection{Platooning objectives}

To create platoons, a platooning service provider can consider different objectives. Here, we discuss two important ones.

\section{Minimize the system-wide fuel cost}

This objective aims to minimize the total fuel cost of all trucks in the system. To determine the net costs of a platoon, one should not only consider the fuel savings that occur within the platoon but also the additional fuel consumed to create the platoon due to detours or speed changes to catch up with a truck. Using a fuel consumption model, Liang et al. (2013) conclude that a platoon is beneficial only if the platooning distance is much larger than the catch-up distance. The minimization of the fuel costs would initially be one of the main operational benefit of platooning for individual truck operators.

By minimizing the fuel costs, we also implicitly increase some of the societal benefits of platooning. Minimizing the fuel consumption is equivalent to minimizing the emissions (Scora and Barth, 2006). Also, when we minimize the fuel costs, longer platoons are preferred as the total savings will be higher with more following trucks in the system. Such longer platoons are associated with more efficient road utilization since the trucks within a platoon drive closer together.

Note that minimizing fuel costs does not necessarily reduce traffic congestion as it may increase the number of trucks on a specific road. On the one hand, the reduced space utilization as a result of platooning might help improve the traffic throughput. On the other hand, when too many trucks take the same road in an effort to form platoons, congestion levels may rise.

\section{Maximize the number of trucks in a platoon}

Instead of minimizing the system-wide fuel cost, a platoon service provider could also maximize the number of matched trucks in the system. The increased likelihood of finding a platoon may be an important criterion to keep truck companies involved. Furthermore, 
involving more companies by creating more matches in the initial stages of technology deployment could help spread confidence and trust in the system. The higher matching rate could consequently stimulate larger participant pools by attracting more truck companies in the future. Moreover, the possibly higher number of platoons created as a result of this objective may help gain more experience with automatic driving and platooning which could prove to be invaluable for the future success of the system. Note that this objective could potentially conflict with the previously described objective of minimizing the fuel costs.

\subsection{Constraints on platoon formation}

Various prerequisites determine whether it is feasible to form a platoon between a set of trucks. One of the most important constraints is the timing of the trips. Since freight transportation typically operates within tight time windows that are specified by the customers, there may be only little flexibility to wait for another truck to form a platoon. Instead of hard constraints on the time windows, table 1 shows that some studies consider soft time constraints by penalizing delays.

Besides the customer imposed time windows, platoons also have to abide by driving time regulations (Goel, 2014; Goel et al., 2012; Goel and Rousseau, 2012; Goel, 2010). These regulations dictate specific time periods in which trucks need to take breaks. Incompatible break times may render certain platoons infeasible.

Furthermore, it may not be possible to form platoons between certain types of trucks. The platooning technologies of different truck manufacturers are currently incompatible, so it is only possible to form platoons with trucks of the same brand (Brizzolara and Toth, 2016; Berger, 2016). Also, the nature of the load (for example, dangerous goods) may preclude a truck from being part of a platoon (Meisen et al., 2008).

It is likely that there will be legal limitations on the length of the platoon (Eckhardt et al., 2016). This is to prevent long platoons disturbing the traffic flow by making it difficult for other vehicles to merge onto highways. Additionally, long platoons could lead to increased wear and tear of roads and bridges since such infrastructure has not been designed for dense truck platoons. Also, the required communication technology for platooning may not be very reliable in tunnels. As a result, it may become necessary to construct dedicated 
infrastructure for platoon traffic. This type of routing constraints are considered in the area of convoy planning (see Tuson and Harrison (2005); Kumar and Narendran (2010); Tuson and Harrison (2005); Chardaire et al. (2005) for an example).

Apart from these technical and operational constraints, personal and inter-organizational considerations may also play an important role in platoon formation. That is, not all companies would be willing to platoon with each other because of concerns related to trust or competition. Due to these issues, some companies may only want to form platoons within their own fleets or within a restricted coalition of fleets.

Next to restrictions on which trucks can form platoons together, there may be restrictions on the platoon sequence. That is, loading weights, torque ratings and the brake capacity determine safe possible truck sequences in a platoon (TNO, 2016). For example, trucks should be arranged in ascending order of their engine power to mass ratio. This is to ensure that the leading trucks do not pull away on uphill terrain (Nowakowski et al., 2015). Safety is another important consideration. To prevent collisions, for example, the truck with the worst braking performance should drive in front.

Table 1 provides an overview of the different objectives, constraints, and the decision variables that are used in the platooning literature.

\subsection{Dividing the benefits of platooning}

Trucks that participate in a platoon directly benefit from lower fuel consumption. However, these savings depend on the position in the platoon as there are more savings for the following truck(s) than for the leading truck. Moreover, trucks may incur different costs, such as detour costs, to join the platoon. This means that it may be necessary to redistribute the total system-wide benefits among the different participants in a platoon. In determining the total benefits, one should not only consider the benefits within the platoon but also the costs associated with forming the platoon such as the detour and waiting costs.

With scheduled truck platoons, simple proportional rules may be an appropriate way to divide the total system-wide benefits. However, this becomes difficult in a dynamic setting in which trucks can join and leave a platoon at any time. This is related to the division of shared benefits in multi-passenger ride-sharing (Furuhata et al., 2013). This issue is related to the 
stream of literature on inter-organizational collaboration (Cruijssen et al., 2007). Much of the research in this area makes use of cooperative game theory which considers scenarios where different parties form alliances that aim to achieve some goals jointly in an attempt to increase their individual profits (see Elkind and Rothe (2016) for more information). Cooperative game theory has been extensively applied to solve various benefit allocation problems in the area of logistics. For examples of applications, see Lozano et al. (2013); Frisk et al. (2010); Krajewska et al. (2008). For a review of studies, see Guajardo and Rönnqvist (2016).

If the same trucks regularly platoon together as in a coalition, they could share the benefits by taking turns as leading truck. Richert and Cortés (2012) propose a similar idea in the context of unmanned aerial vehicles (UAVs). This is also comparable to carpool schemes in which different participants act as driver each time, e.g., Fagin and Williams (1983) propose scheduling algorithms to determine which carpool participant should drive in each carpool to fairly divide the workload. Likewise, one could assign the more beneficial following positions to the truck that incurs most costs to join the platoon.

Another consideration in the centralized planning of platoons is that system-optimal solutions are not necessarily optimal for each of the individual participants. A solution is not 'stable' if there exist pairs of trucks that are better off forming a platoon together than with the system assigned platoon partners. Wang et al. (2017) consider this in the context of dynamic ride-sharing.

It may be difficult to establish system-optimal solutions due to strategic behaviour of the participants. To maximize their own benefits, companies or drivers may reveal false information if it means they find a better match.

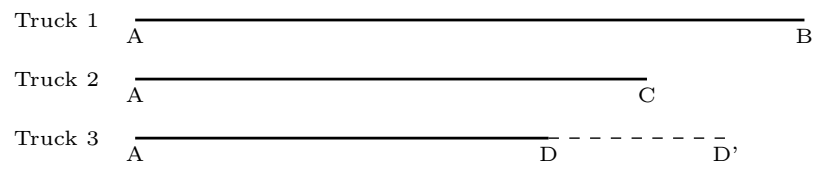

Figure 2: Example of strategic behaviour by truck 3

A simple example is shown in figure 2. Here, trucks 1,2, and 3 need to travel from A to

B, C, and D respectively. The system-optimal solution would entail a platoon with trucks 
Table 1: Overview of platoon planning literature

\begin{tabular}{|c|c|c|c|c|c|c|c|c|c|c|c|c|c|}
\hline \multirow[t]{2}{*}{ Author(Year) } & \multicolumn{2}{|c|}{ Objective } & \multicolumn{4}{|c|}{ Constraints } & \multicolumn{4}{|c|}{ Decisions } & \multicolumn{3}{|c|}{ Dynamics } \\
\hline & Fuel & Delays & Timing & Speed $^{1}$ & Detour & Length & $\mathrm{PC}$ & $\mathrm{R}$ & $\mathrm{S}$ & $\mathrm{SC}$ & Scheduled & Real-time & Opportunistic \\
\hline Sokolov et al. (2017) & $\bullet$ & & - & & • & & $\bullet$ & $\bullet$ & $\bullet$ & & $\bullet$ & & $\bullet$ \\
\hline Zhang et al. (2017) & - & - & $\bullet$ & & & & $\bullet$ & & $\bullet$ & & $\bullet$ & & \\
\hline Adler et al. (2016) & $\bullet$ & $\bullet$ & $\bullet$ & & & - & $\bullet$ & & & & & $\bullet$ & \\
\hline Larson et al. (2016) & $\bullet$ & & $\bullet$ & & • & & $\bullet$ & $\bullet$ & $\bullet$ & & $\bullet$ & & \\
\hline Liang et al. (2016) & $\bullet$ & & - & $\bullet$ & & & $\bullet$ & & & $\bullet$ & & $\bullet$ & \\
\hline Van de Hoef (2016) & $\bullet$ & & $\bullet$ & $\bullet$ & & & $\bullet$ & & $\bullet$ & $\bullet$ & $\bullet$ & $\bullet$ & \\
\hline Nourmohammadzadeh & $\bullet$ & & $\bullet$ & & & & $\bullet$ & $\bullet$ & $\bullet$ & & $\bullet$ & & \\
\hline Hartmann & & & & & & & & & & & & & \\
\hline$(2016)$ & & & & & & & & & & & & & \\
\hline Larsson et al. (2015) & $\bullet$ & & $\bullet$ & & & & - & $\bullet$ & $\bullet$ & & $\bullet$ & & \\
\hline Liang et al. (2014) & $\bullet$ & & $\bullet$ & $\bullet$ & & & $\bullet$ & & $\bullet$ & $\bullet$ & $\bullet$ & & $\bullet$ \\
\hline Liang et al. (2013) & $\bullet$ & & & $\bullet$ & • & & $\bullet$ & & & $\bullet$ & & - & \\
\hline Larson et al. (2013) & $\bullet$ & & $\bullet$ & $\bullet$ & & & $\bullet$ & $\bullet$ & & $\bullet$ & $\bullet$ & $\bullet$ & \\
\hline Meisen et al. (2008) & $\bullet$ & $\bullet$ & $\bullet$ & & & $\bullet$ & $\bullet$ & & & & $\bullet$ & & \\
\hline
\end{tabular}

PC - platoon composition, $\mathrm{R}$ - route, $\mathrm{S}$ - schedule, $\mathrm{SC}$ - speed changes

1 - studies without a speed constraint assume a fixed driving speed

1 and 2 since the overlap in their routes is the longest. Knowing this, truck 3 could falsely report his destination as D' resulting in a platoon with trucks 1 and 3. Clearly, this isn't the system-optimal solution any more.

To prevent strategic behaviour, one may use formal agreements or contracts (see Schwartz 
between platooning and freight consolidation and ride-sharing.

\subsection{Freight consolidation}

Freight consolidation is used as a means to facilitate more efficient and frequent shipping

\subsection{Ride-sharing}

Ride-sharing is the practice of sharing rides as a means of reducing congestion, pollution and fuel costs by using empty seats of passenger cars. The rationale behind ride-sharing is similar to that of freight consolidation as discussed above. Similar to load being consolidated 
consolidation is that people are less flexible than load specially with respect to travel and waiting times.

Traditionally, ride-sharing or carpooling was used by people regularly travelling to the same place at the same time (Levofsky and Greenberg, 2001). In today's world, ride-sharing systems exist that dynamically match riders with drivers, in real-time, based on their locations and times.

In ride-sharing, drivers are matched with riders that need to be picked up at their origins and dropped off at their destinations. Similar to freight consolidation, the pick up and drop off locations are fixed at the origin and destination. The service time is determined by the time it takes for this pick up and drop off to be executed.

Truck platooning and ride-sharing may result in less fuel consumption and consequently, reduced emissions. They involve matching entities with similar routes and time schedules. Both of them operate under certain capacity constraints. For ride-sharing, the capacity is associated with the number of seats in the car. For platoons, the length of a platoon is conceptually unlimited but will likely be restricted by legislation. Also like in platooning, drivers part of ride-sharing services are likely to have to make some detours or adjust their time schedules to pick up riders.

Ride-sharing has recently started to receive much attention in literature. Furuhata et al. (2013) and Agatz et al. (2012) provide reviews of the different studies and the various solution methodologies in ride-sharing literature. Similar concepts have also been applied to freight (see Arslan et al. (2016)). Table 2 summarizes the comparison between platooning, ridesharing and freight consolidation. 
Table 2: Comparison of platooning with ride-sharing and freight consolidation

\begin{tabular}{|c|c|c|c|}
\hline & Truck platooning & Ride-sharing & Freight consolidation \\
\hline \multicolumn{4}{|l|}{ Supply } \\
\hline Entity & Trucks & Drivers & Trucks \\
\hline Capacity & Allowed maximum platoon size & Vehicle capacity (no of passengers) & Load weight and volume limits \\
\hline \multicolumn{4}{|l|}{ Demand } \\
\hline Entity & Trucks & Riders & Load \\
\hline Location & Flexible & Fixed & Fixed \\
\hline Service time & Negligible & Pick up and drop off time & Loading and unloading time \\
\hline Service quality & Detour, excess travel time, success rate & Detour, excess travel time, success rate & Detour, safety, reliability \\
\hline \multicolumn{4}{|l|}{ Benefits } \\
\hline Individual & Reduced labour and fuel costs & Reduced costs due to shared capacity & Reduced costs due to economies of scale \\
\hline Societal & Reduced emissions, road utilization & Reduced emissions, road utilization & Reduced emissions, road utilization \\
\hline
\end{tabular}




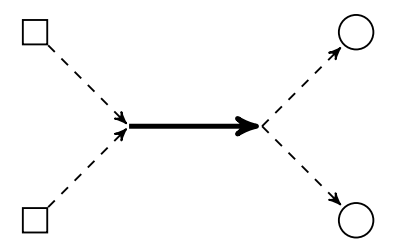

(a)

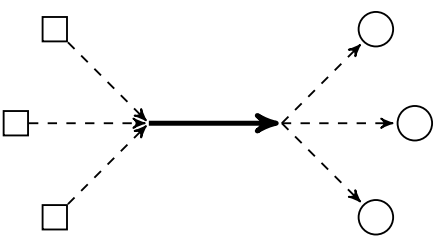

(b)

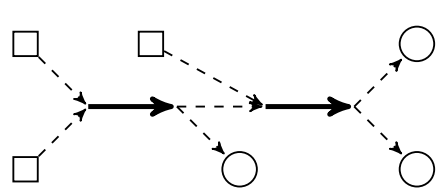

(c)

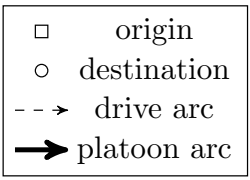

platoon arc

Figure 3: Examples of restricted platoon settings - (a) a two truck platoon - single platoon per trip, (b) a three truck platoon - single platoon per trip, and (c) a two truck platoon - multiple platoons per trip

\section{Scheduled platoon planning}

In this section, we classify different platoon planning problems and discuss the relevant

\subsection{Unrestricted platooning}

In the unrestricted setting, a truck may join and leave a platoon en route at any point in time. Moreover, there are no restrictions on the number of trucks in a platoon. A simple example of such a scenario with four trucks is depicted in figure 4. 

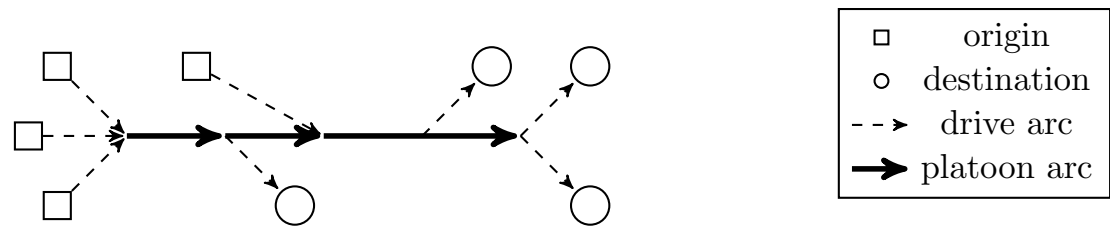

Figure 4: Unrestricted platooning - trucks allowed to join and leave a platoon at any instant

\subsubsection{Unrestricted platooning - fixed routes} the savings per pair of trucks, they use a fuel model which considers the speeds in addition to topographic and vehicle characteristics. In this study, they allow the lead vehicle to slow 
down in addition to the following vehicle speeding up to form platoons and conclude that it is more efficient.

Zhang et al. (2017) focus on the sub problem of determining feasibility of a pair of trucks by studying the platoon coordination problem under travel time uncertainty. As part of a cost minimization framework, they focus on determining the savings for a pair of trucks considering fuel related costs, travel-time related costs, and penalties for deviating from the planned schedule. When both trucks follow the same path, they observe that above a certain threshold in the waiting time, the vehicles are better off driving alone since the penalties outweigh the fuel savings. They extend their analysis to converging and diverging routes and find that platooning on converging routes is less beneficial due to the extra costs of waiting at the merging point on the network.

As we explained earlier in section 2.2, having too many trucks on the same road to maximize the platooning potential could cause congestion and be detrimental to the system. This will occur if the number of trucks exceeds the capacity of a link. The area of traffic flow research could be used to look into this aspect. Traffic assignment models aim to minimize travel time in the system by assigning vehicles to specific links in the network. For examples, see Angelelli et al. (2016), Merchant and Nemhauser (1978). Using this line of reasoning, Farokhi and Johansson $(2013,2014)$ consider vehicles that decide their time of travel based on their preferred time, average traffic velocity and the congestion tax at that time. Trucks that can platoon have an additional incentive use the road at the same time as other trucks. They model the problem as a congestion game and use game theoretic concepts to study how the traffic flow and platooning incentives interact. One of their observations is that if there are more fuel savings associated with platooning, more trucks start travelling in similar time intervals. In this study, the authors consider a single stretch of road and therefore, the routing element isn't involved.

\subsubsection{Unrestricted platooning - flexible routes}

In this setting, the platoon planning problem includes determining the routes of each truck in addition to the departure times and/or speeds. The additional routing decisions make the problem more difficult to solve. A number of studies in the literature consider this 
problem.

Larson et al. (2013) consider a simplified version of the problem in which local controllers 
Larson et al. (2016) also adopt a system-wide perspective and formulate a mixed integer programming model with the objective of minimizing the amount of fuel used. The trucks are allowed to wait to form platoons. They include certain additional constraints to reduce

redundant variables and hence, the problem size. They perform experiments on a simple grid network and a representation of the Chicago highway network for instances with 25 trucks.

Nourmohammadzadeh and Hartmann (2016) propose heuristics based on a genetic algorithm. On comparison with the optimal solutions for smaller instances of about ten trucks, the genetic algorithm exhibits similar levels of performance at faster speeds. For larger instances of 30-50 trucks where the solver is inadequate, the genetic algorithm still generates fuel saving results in under a minute. The trucks are routed on a network that includes 20 major German cities.

The area of convoy planning provides some parallels to this platoon setting. This is primarily used in military applications where safety and therefore, time are crucial considerations. The study by Valdés et al. (2011) can be seen as a special case where a transportation unit in a city needs to merge with a convoy that is travelling in a circular path across the city. They use dynamic programming to route the transportation unit to the convoy in an efficient way. The synchronized arc routing problem (Salazar-Aguilar et al., 2011) also shares some characteristics with the platoon planning problem. This problem also involves synchronizing the routes of multiple vehicles. Salazar-Aguilar et al. (2011) propose a local search heuristic to solve this NP-hard problem for large instances.

\subsection{Restricted platooning}

Section 4.1 presents several studies that address different variants of the unrestricted platoon planning problem. It is, however, not clear if the solution approaches developed for these unrestricted settings would also be effective in the more restricted settings. Certain restrictions may be exploited by making use of specialized algorithms. For instance, Agatz et al. (2011) are able to solve large instances of the ride-sharing problem to optimality by restricting the number of riders per ride-share match. Nevertheless, the introduction of restrictions may also complicate matters as it makes it more difficult to find feasible solutions.

\subsubsection{Restricted platooning - fixed routes}


This setting also represents a scheduling problem where we need to determine the departure times/speeds of trucks given predetermined routes. Here, restrictions such as the ones relating to the platoon size or number of platoons per trip (see figure 3) need to be taken into account.

We are aware of one study that explicitly considers this setting. Meisen et al. (2008) aim to find profitable truck platoons given a set of routes. They set the maximum size of the platoon to be either two or four. To determine if a platoon is profitable (has net savings), they consider multiple criteria such as common distance, waiting time, fuel consumption etc. They propose a data mining based heuristic to solve this problem. The trucks are first categorized based on characteristics such as their load. Among these trucks, grouping possibilities are determined based on the trucks' physical characteristics. Within these trucks that are grouped together, platoons are planned based on the overlap in the routes. In addition to the fuel costs, the costs associated with waiting are also considered. To test the algorithm, the authors use synthetic datasets with up to 5000 routes and are able to find profitable platoons. To limit the exponential growth in the number of profitable platoons with an increase in the number of routes, they set limits on the waiting time, common distance and profit per platoon.

Other areas of research provide parallels to this setting. For example, Dumas et al. (1990) for an example of a fixed route scheduling problem. Also, the route overlaps may also be found based on algorithms used for solving the well-known longest common subsequence problem in computer science which, as the name suggests, involves finding the subsequence of maximum length that occurs in a set of given sequences (see Iliopoulos and Rahman (2008) for more information).

If we aim to reduce the inconvenience or waiting times for the trucks to form platoons, this setting resembles the area of schedule synchronization in public transit (see Wong et al. (2008); Daduna and Voß (1995); Voß (1990); Domschke (1989) for examples).

\subsubsection{Restricted platooning - flexible routes}

In this setting, we need to determine the routes of the trucks in addition to the schedules/ speeds of the trucks while taking different restrictions into account. While this platoon 

reach the meeting point. This incorporation of movement of the riders strengthens the link to platooning where both the entities i.e. trucks that need to be matched are moving.

Ride-sharing is a special case of the well-known general pick up and delivery problem. 
The general pick up and delivery problem (Savelsbergh and Sol, 1995) considers vehicles that need to fulfil transportation requests by picking up goods at their origins and delivering them to their destinations. This can be extended to truck platooning where instead of goods, trucks need to be "picked up". Unlike the traditional pick-up and delivery problem, there are no fixed start and end locations defined as both vehicles are mobile. This is linked to the vehicle routing problem with roaming delivery locations. This problem considers the deliveries of shipments to the trunk of a customer's car (Reyes et al., 2016).

Conceptually similar problems are also seen in multi-modal freight transportation. Heeswijk et al. (2016) consider inter-modal networks with terminals where freight coming in by truck is consolidated and moved to a different terminal by barge, rail or truck from where the freight is sent to its destination. If we consider a special case where all the trucks are headed to the same destination, the problem is linked to the merge in transit method of operation seen in parcel deliveries (see Croxton et al. (2003)).

Furthermore, the problem is related to the area of vehicle routing with synchronization constraints. A survey of problems and related solution methods in this area may be found in Drexl (2012). In this area of problems, there is an interdependence in the routes of different trucks. Of the different types of synchronization described by Drexl (2012), operation synchronization is specially relevant for truck platooning. Operation synchronization refers to the spatial and temporal offsets allowed for different trucks to begin certain tasks, for example, depart from a depot. This is directly related to the route and time flexibilities.

Table 4 provides an overview of the methods and instances considered by the various studies.

\section{Levels of human involvement in platooning}

Up to now, we have considered platoons between trucks that require fully engaged drivers. As technology develops and legislation permits, driverless platoons may become possible in the future. The required level of human involvement is likely to gradually decrease as automated driving technology evolves. Since the widely-used SAE levels of driving automation (SAE International, 2016) focus on individual vehicles and do not consider platooning, we propose the following new classification to describe different levels of human involvement in 
Table 3: Classification of platooning literature

\begin{tabular}{l|l|l} 
& Flexible routes & Fixed routes \\
\hline No restrictions on platoons & Sokolov et al. (2017) & Zhang et al. $(2017)^{*}$ \\
& Nourmohammadzadeh and Hartmann (2016) & Liang et al. (2016) \\
& Larson et al. (2016) & Van de Hoef (2016) \\
& Larsson et al. (2015) & Liang et al. (2014) \\
\hline Restrictions on platoons & Larson et al. (2013) & Meisen et al. (2008) \\
special case/ sub-problem & &
\end{tabular}

platooning.

- Human driven platooning with in-platoon resting

- Hybrid platooning

- Driverless platooning

These different types of platooning give rise to new planning problems that we discuss in this section.

\subsection{Human driven platooning with in-platoon resting}

In this type of platooning, the following trucks can handle all the driving tasks which means that the drivers may rest while being in the truck. Therefore, this setting allows for more efficient utilization of the drivers. For this to be implemented in practice requires both technological feasibility and legal clearance.

Legal stipulations also control the amount of time for which a driver is allowed to drive. That is, truck drivers in most countries are subject to limits on their driving times before being required to take a break. The regulations depend on the type of vehicle and the country. For example, EU regulations state that a driver must take breaks totalling at least 45 minutes after a maximum of 4.5 hours of driving (Government-UK, 2016). Similarly, in the US, a break of 30 minutes is required after at most eight hours after a driver begins his duty (Goel, 2014). Regulations typically also prescribe weekly limits in addition to the limitations within a day. See Goel (2014); Goel et al. (2012); Goel and Rousseau (2012); 

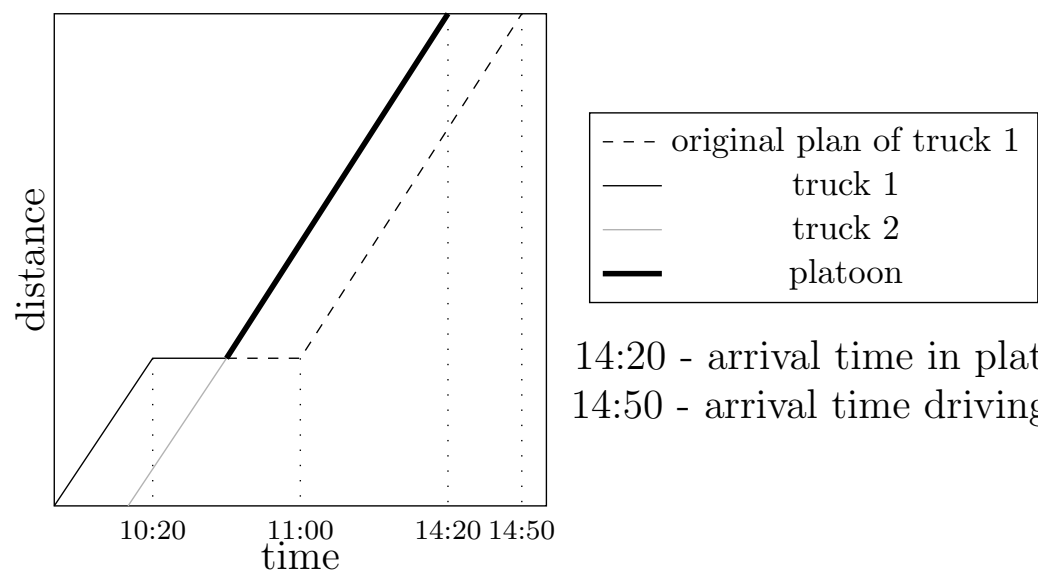

14:20 - arrival time in platoon

14:50 - arrival time driving alone

Figure 5: Increased productivity when the driver of truck 1 can take a break in a platoon

Goel (2010) for a detailed overview of different regulations. In this setting, the time as the follower in the platoon would not count as formal driving time.

Allowing breaks during a platoon will relax break-time constraints and as such improve the overall transport efficiency. In particular, it may help increase the productivity of the drivers as they can cover more distance in the same time. Figure 5 illustrates the benefits of being able to take a break within the platoon. The example considers two trucks that share a portion of their route. Truck 1 starts a 40 minute break at 10:00. Ten minutes into his break, truck 2 arrives at the location. Instead of waiting to finish his break, the driver of truck 1 can now finish the last 30 minutes of his break as a following truck of the platoon. This implies that truck 1 now arrives at its destination at 14:20 instead of 14:50. Therefore, there is an improvement in the transit time and associated costs.

The example described in figure 5 considers a two truck platoon where each truck is part of only one platoon in its trip. The same idea can fairly easily be extended when we consider platoons of more than two trucks. Just like the example, the change will affect only the trucks in that platoon. It is, however, more complicated when a truck may be part of multiple platoons per trip. Since the different platoons in a trip are interdependent, any change made to the break of one truck will affect all the platoons in the trip of that truck and, in turn, all the platoons in the trips of the trucks part of the said affected platoons and so on. Therefore, the problem's computational complexity grows rapidly.

At the same time, there is an additional layer of complexity that needs to be considered 
in planning the platoons. Since breaks are allowed only for the drivers in the following trucks, the position of a truck in a platoon becomes an important planning decision. For

longer trips, we could switch the order periodically so that all drivers get an opportunity to take a break. To plan the timing of switches in the platoon sequence, the timing of breaks, and travel times need to be considered. In these settings, time-related costs will probably be more relevant than fuel related costs. As a result, we may see an increase in the total system-wide travel distance which are compensated for by the savings in time.

\subsection{Hybrid platooning}

In this type of platooning, only the leading truck requires a human driver and the following trucks can be driverless. This means that the driver of the following truck is no longer required for parts of the trip, which may lead to labour cost savings (Kilcarr, 2016). Unless the leading and following truck have exactly the same itinerary (and following trucks basically serve as trailers), the following trucks would still need drivers for the first and last part of their trips when they are driving alone. As a result, the formation or dissolution of platoons requires specific points and can no longer be done en-route. Drivers could be moved between these points by taxi. The planning of these taxi rides gives rise to a pick-up and delivery problem of drivers.

The pick-up and delivery problem is a well known optimization problem, see for example, Berbeglia et al. (2010); Savelsbergh and Sol (1995). A special case of pickup and deliveries that involves people is referred to as the Dial-a-Ride Problem (Cordeau and Laporte, 2007). This is also similar to the last/first mile problem seen in scheduled public transit. The first mile problem is similar to the car pooling problem where multiple users are picked up and transported to a common flexible destination which could be any point on a public transportation line (see Minett (2013)). The last mile problem considers the opposite scenario where people are picked up from the public transportation line and taken to their destinations (see Wang and Odoni (2014); Cheng et al. (2012)).

Hybrid platooning also gives rise to new opportunities with regards to driver roles. The drivers may have different duties such as a platoon leader or a last mile driver. Furthermore, during the pick up and delivery of drivers, drivers may swap roles. The driver dropped off 
may take over as the leading driver of the platoon while original platoon leading driver may take over the decoupled truck and complete its final leg. This is specially relevant for the planning of shifts and breaks. This is linked to the general area of crew scheduling (see Ernst

et al. (2004); Raff (1983)). Vehicle routing and crew scheduling has been done in parallel in most of the literature (see Hollis et al. (2006) for an overview). Drexl (2011) mentions that the truck and trailer approach could also be used in this way. The drivers may be considered vehicles which can couple with a truck that is left at intermediate locations.

Apart from being picked up by a driver, a truck could also be picked up by another platoon. Also, drivers could pick up trucks from one location and leave it at another location where the truck is then picked up by another platoon. These two basic arrangements may be combined in any order and form. Therefore, there is quite some flexibility in the planning of hybrid platoons. On that account, following trucks can essentially act as trailers that need to be picked up and dropped off. Therefore, this problem is related to the the truck and trailer problem (see Derigs et al. (2013); Villegas et al. (2013); Chao (2002)) and the swap body vehicle routing problem (see Lum et al. (2015); Absi et al. (2015); Miranda-Bront et al. (2015); Huber and Geiger (2014)). Meisel and Kopfer (2014); Drexl (2011) categorize transport means as active and passive. Active means are also allowed to transfer load onto passive means in addition to picking them up. In this platooning context, a truck could either be active or passive depending on its use at a particular instant.

\subsection{Driverless platooning}

This form of platooning involves completely driverless trucks which provides a greater degree of flexibility since the platoons do not have human drivers that need to go home or take breaks. The planning of driverless platoons is similar to the planning of regular human-driven platoons but the additional flexibility creates more room for optimization. For instance, the absence of drivers means that trucks do not have to return to a fixed location. This means that the truck could go to a different depot than its starting depot if it results in more platooning opportunities now or on the next trip.

\section{Real-time platoon planning}


In our discussion up to now, we assumed that all the information required for planning the platoons is known in advance and accurate. However, in practice, trucks may continuously arrive and withdraw from the platoon planning system thereby changing the nature of information.

The dynamics increases the complexity of the decision making process. It requires realtime information of the states of all vehicles and communication methods to inform the vehicles of any changes. Decisions need to be made quickly as trucks are moving so platooning opportunities at one point in time may no longer be available at a later point in time. This dynamism links this problem to the area of dynamic vehicle routing in which route plans may be adjusted when new information becomes available (see Pillac et al. (2013) for an overview).

Dynamic planning could be carried out in a time-based or an event-based manner (see Agatz et al. (2011)). A well known example of a time-based approach is the rolling horizon approach where optimization is repeated after a given time interval. Instead of the optimization being repeated after a certain time, it also could be triggered by an event such as the arrival of a new entity as the system. This is the case with event-based planning and is considered by Van de Hoef (2016) and Larson et al. (2013) who repeat the optimization when new information becomes available.

Based on historical information, we may have some probabilistic information about future events. For instance, historical data could provide an indication about trip announcements from freight transporters. For instance, if it is known that some trucks traverse the same route fairly regularly, there is a high probability of them being able to form platoons.

Adler et al. (2016) look at a case of real-time platooning of multi truck platoons from a queueing perspective by considering historical data to model the arrival of trucks. They consider a Poisson-distributed series of vehicles arriving at a particular station and all headed towards a common destination. The vehicles form platoons at this station and drive together as a single platoon to the common destination. Therefore, they allow the formation of multi truck platoons. Vehicles can wait for each other at the station but this will increase the delay. They look at this trade-off between energy savings and delay. Two sets of platoon formation policies are defined - all the trucks at the station leave as a platoon when either 
a certain time period has elapsed or a threshold platoon size is reached. On comparing these two sets of policies, they observe that the performance is dependent on the size of the platoons produced. The threshold platoon size policies allow an average of one truck more per platoon than the time table policies. As a consequence, the threshold policies perform better in terms of the energy saved for a given delay.

Moreover, the stochasticity of information links the problem to the area of stochastic vehicle routing (Gendreau et al., 1996). See for example, Bent and van Hentenryck (2004); Powell (1996) for the usage of stochastic information in dynamic settings. Furthermore, the travel times of trucks could be uncertain due to the weather, traffic amongst other factors (van Lint et al., 2008). For examples with stochastic travel times, see Kenyon and Morton (2003); Laporte et al. (1992). This was considered by Zhang et al. (2017) as described in Section 4.2.1.

\section{Vehicle routing with platooning}

Instead of planning only single trips from an origin to a destination, one could also consider platooning opportunities for routes with multiple stops. If there is flexibility in the sequence of stops, there is more room to form platoons.

Figure 6 shows an example in which the number of platooning opportunities may be increased by changing the sequence of stops. The figure shows the routes of two trucks with their stop sequences. Initially, there is no common path in their routes and, therefore, no opportunities to platoon. Switching the order of stops $c$ and $d$ on route 1 gives rise to a platooning opportunity between stops $b$ and $d$.

This combines platoon planning with vehicle routing. There is a large body of research on solution approaches for the vehicle routing problem. Laporte (1992) provides an overview of these different approaches. In the usual variant of the problem, the loads need to be assigned to vehicles subject to the capacity constraints. The trucks could operate out of a single depot or multiple depots (for examples of the multi depot setting, see Crevier et al. (2007); Lim and Wang (2005); Cordeau et al. (1997); Renaud et al. (1996)).

When all trucks belong to the same fleet or company, the problem can be solved by considering a vehicle routing problem that incorporates platooning opportunities. This is 


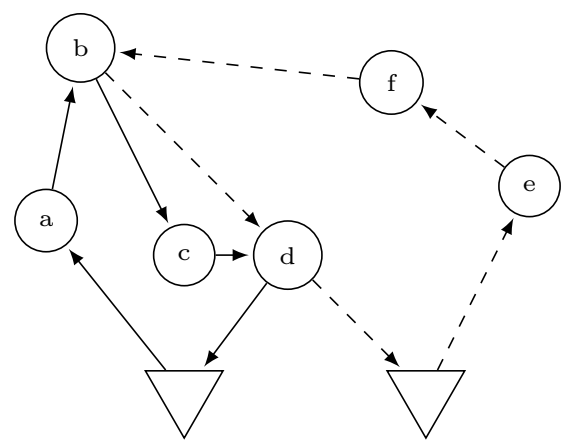

No platooning opportunity
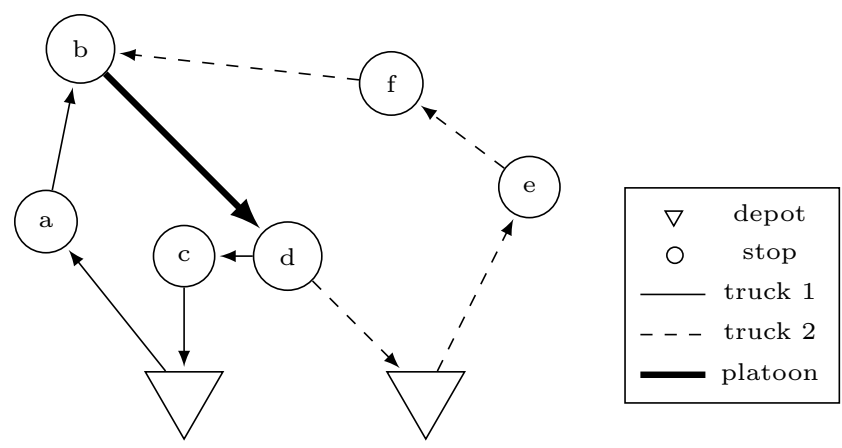

Platooning opportunity between stops $b$ and $d$

Figure 6: Changing the sequence of stops to create a platooning opportunity

different from the standard vehicle routing problem since the routing overlap creates pla-

tooning opportunities. Instead of just the shortest path, we need to consider multiple paths between a pair of points to maximize the platooning opportunities.

When multiple fleets are involved, there may be practical restrictions on the assignments of vehicles to stops. That is, the stops allocated to each fleet are known in advance and we have fixed clusters. This means that the route assignments are fixed and there is flexibility in the sequence of stops. This links the problem to the area of cluster first, route second vehicle routing literature (see Prins et al. (2014) for an overview). Here, the customers are clustered together and the customers in a cluster are visited by the same truck. The routing phase can be treated as solving a travelling salesman problem with time windows within each cluster (Laporte et al., 2000).

\section{Network design and platooning}

The previous section described how platooning could have an impact on the way that freight is routed through the network at the operational level. Platooning could impact at strategic and tactical level decisions as well.

The strategic level decisions involve long term aspects such as physical network design (Crainic, 2000). For instance, parts of the network may be heavily used by platoons and require infrastructural changes such as reinforcement of roads, new lanes, additional communication support in tunnels etc. A similar rationale is used in the context of automated vehicles by Chen et al. (2017, 2016). Also, since the starting locations of trucks influence 


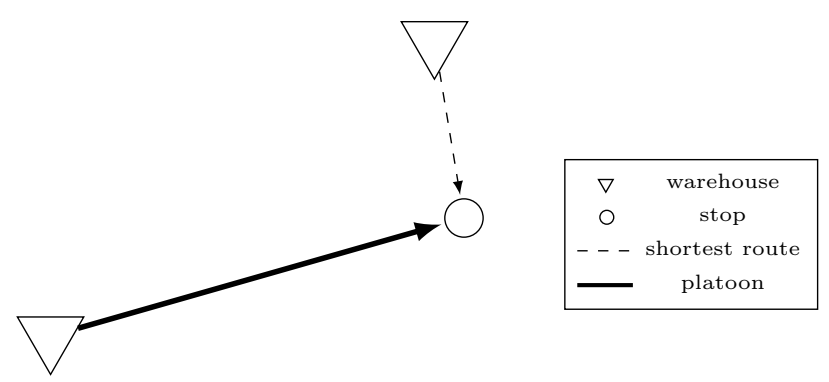

Figure 7: Change in order assignments because of platooning

platooning opportunities, there is an incentive to move facilities such as warehouses and

depots closer to each other. This relates to the concept of economies of agglomeration (see Glaeser (2007)).

Tactical level decisions relate to service network design which involves decisions mainly pertaining to the organization of services, and the routing of freight. Crainic (2000) provides an overview of research in this area. Because of increased platooning opportunities, certain orders may be assigned to different warehouses than the ones closest to the shipment location. Figure 7 shows a simple example of such a situation. The flexibility of platooning means that this decision should be made as late as possible based on real-time platooning opportunities on the different routes. Xu et al. (2009) look at a similar problem in the e-commerce setting citing the dynamic nature of the system. This dynamic nature also creates a link with the dynamic service network design problem (see Dall'Orto et al. (2006)). Additionally, we can view the flexible formation of a platoon as an instantaneous switch in the transportation mode. This links platooning to the multimodal network design problem (for an example, see Yamada et al. (2009)).

\section{Conclusions and future research}

Truck platooning can be seen as the first step towards automated driving in an open environment. Truck platooning has the potential to provide cost savings and is associated with several societal benefits. To efficiently reap the benefits of platooning requires appropriate planning and optimization approaches.

This paper outlined various planning challenges encountered in platoon planning. Also, the paper provided an overview of relevant operations research models from different areas. 


\begin{tabular}{l|l|l|l}
\multicolumn{2}{|c}{ Table 4: Overview of platoon planning literature } \\
Author(Year) & Solution method & Network used & Largest instance size \\
\hline Zhang et al. (2017) & Exact & Artificial - single road, junction \\
Sokolov et al. (2017) & Exact & Artificial - 10 X 10 grid \\
Larson et al. (2016) & Artificial - 10 X 10 grid, Real-life - Chicago highway network & 25 \\
Liang et al. (2016) & Heuristic - local search & Real-life - single Swedish highway \\
Van de Hoef (2016) & Exact, Heuristic - local search & Real-life - Swedish mainland \\
Nourmohammadzadeh and Hartmann (2016) & Exact, Heuristic - genetic algorithm & Real-life - Twenty German cities \\
Adler et al. (2016) & Analytical - queueing theory & Artificial - single road \\
Larsson et al. (2015) & Exact, Heuristic - local search & Real-life - German autobahn network \\
Liang et al. (2014) & Real-life - region in Europe \\
Liang et al. (2013) & Analytical - fuel model & Artificial - single highway \\
Larson et al. (2013) & Real-life - German autobahn network \\
Meisen et al. (2008) & Heuristic - truck platoon sequential & Real-life - Two German zip codes \\
& pattern algorithm (based on pattern & N/A * \\
& growth) & 1800 \\
\end{tabular}

*use an arrival rate of trucks

Table 4 provides an overview of the platoon planning literature discussed in this paper. From tables 1,3 , and 4, we see that there are several papers that address various problems but approaches to deal with the dynamic, advanced, and restricted forms of platooning etc. are still lacking. More specifically, the areas for future research include -

Optimization. All studies acknowledge that the different platoon planning problems are difficult to solve. Table 4 shows that most studies resort to similar kinds of basic local search heuristics on relatively small problems. One interesting direction for future research is to study the effectiveness of more sophisticated meta-heuristics (see Cordeau et al. (2002); Bräysy and Gendreau (2005a,b)) such as tabu search and simulated annealing in solving real-life platoon planning problems. To allow the systematic comparison of the different solution approaches, we would require the introduction of standard benchmark instances.

Another interesting direction for future research is the development of specialized solution methods to handle the various restrictions such as those relating to platoon size. Given that these restricted settings are the most likely to occur in reality especially in the initial phases of deployment, research in the area is required.

Higher levels of automation reduce the level of human involvement and therefore open up a set of new planning problems. We are not aware of any research in this area so far.

Dealing with uncertainty. Areas relating to real time planning are of interest. In practice, the information continuously changes with trucks entering and leaving the system. Also, travel time uncertainty represents a realistic scenario but is more complex to solve 
and implement. Zhang et al. (2017) do study stochastic travel times but in a small setting. Moreover, we are not aware of any research dealing with stochastic and dynamic arrivals of trucks for platooning.

When we consider real-time planning, congestion could also be taken in to account as a result of too many trucks being routed on the same links. A few trucks could be routed differently to increase the benefits for the whole system. Other traffic also needs to be considered when this approach is used. This is conceptually related to the area of traffic assignment and multimodal service network design.

System sustainability. With a very small number of participants, the chances of formation of platoons go down. To ensure that platooning is sustainable in the long run, several 'special' steps might need to be taken during the initial phases of implementation.

From the planning perspective, maximization of the number of companies involved could be a way to go. Incentive schemes from the government to ensure benefits could also play a role. For examples, these incentives could be to subsidize the technology or provide special cost cuts for platoons. The determination of such measures and their effects on the system are interesting areas to look into.

Also, to ensure system sustainability, ways to ensure fair participation and prevent strategic behaviour are necessary. Participants may try to maximize their own profit rather than contributing to the system benefits. Designing mechanisms such as rating systems etc. to prevent this is required.

Network design. Platooning could have implications for the transport and supply chain network designs. As discussed, parts of the transport network may require upgrading to reap the maximum benefits of platooning. Similarly, supply chain network decisions such as locations of facilities might be made differently with platooning in the scene.

These network changes require significant investments and therefore, the expected costs and benefits will have to be carefully weighed against each other. Moreover, as the level of human involvement becomes less, the magnitude of the effects will change due to the additional benefits.

Incentives could again play a role here. Given the societal benefits of platooning, the government may want to encourage some of the (expected) effects so that forming platoons 
becomes easier. Additional research into the effects of platooning on the network will help this cause.

Given the promising potential of platooning, we are optimistic that this review will prompt new research contributions to the area.

\section{Acknowledgements}

We would like to thank Robbert Janssen and Emiel van Eijk (TNO), and Caspar Chorus (TU Delft) for their feedback and comments on earlier versions of the paper.

Funding: This work was supported by the Netherlands Organization for Scientific Research as part of the 'Spatial and Transport impacts of Automated Driving (STAD)' [Project number 438-15-161] and the '2-Truck Platoon matching for collaborative planning' [Project number 438-15-512] projects.

\section{References}

Absi, N., Cattaruzza, D., Feillet, D., Housseman, S., 2015. A relax-and-repair heuristic for the swap-body vehicle routing problem. Ann Oper Res.

Adler, A., Miculescu, D., Karaman, S., 2016. Optimal policies for platooning and ride sharing in autonomy-enabled transportation.

URL http://www . wafr.org/papers/WAFR_2016_paper_110.pdf

Agatz, N., Erera, A., Savelsbergh, M., Wang, X., 2011. Dynamic ride-sharing: a simulation study in metro Atlanta. Transportation Reserch Part B, Methodological 45 (9), $1450-1464$.

Agatz, N., Erera, A., Savelsbergh, M., Wang, X., 2012. Optimization for dynamic ride sharing: A review. European Journal of Operational Research 223 (2), 295-303.

Aivodji, U., Gambs, S., Huguet, M.-J., Killijian, M.-O., 2016. Meeting points in ridesharing: A privacy preserving approach. Transportation Research Part C: Emerging Technologies 72, 239-253. 
Alam, A., Besselink, B., Turri, V., Mårtensson, J., Johansson, K. H., 2015. Heavy-duty vehicle platooning for sustainable freight transportation: A cooperative method to enhance safety and efficiency. IEEE Control Systems 35 (6), 34-56.

Amey, A., 2011. A proposed methodology for estimating rideshare viability within an organization, applied to the MIT community. Annual TRB Meeting 2011.

Angelelli, E., Arsil, I., Morandi, V., Savelsbergh, M., Speranza, M. G., 2016. Proactive route guidance to avoid congestion. Transportation Research Part B.

Arslan, A., Agatz, N., Kroon, L. G., Zuidwijk, R., 2016. Crowdsourced delivery: A dynamic pickup and delivery problem with ad-hoc drivers. SSRN.

Azadeh, K., de Koster, M., Roy, D., 2017. Robotized warehouse systems: Developments and research opportunities. SSRN.

URL https://papers.ssrn.com/sol3/papers.cfm?abstract_id=2977779

Bent, R. W., van Hentenryck, P., 2004. Scenario-based planning for partially dynamic vehicle routing with stochastic customers. Operations Research 52 (6), 977-987.

Berbeglia, G., Cordeau, J., Gribkovskaia, I., Laporte, G., 2007. Static pickup and delivery problems: a classification scheme and survey. Top 15 (1), 1-31.

Berbeglia, G., Cordeau, J.-F., Laporte, G., 2010. Dynamic pick-up and delivery problems. European Journal of Operational Research 202 (1), 8-15.

Berenghem, C., Schladover, S., Coelingh, E., 2012. Overview of platooning systems. In: Proceedings of the 19th ITS World Congress, Oct 22-26, Vienna, Austria (2012).

Berger, A., 2016. Sharing gains and pains - service needs for safe and efficient platooning. Tech. rep., Volvo Group Trucks Technology.

Bräysy, O., Gendreau, M., 2005a. Vehicle routing problem with time windows, part i: Route construction and local search algorithms. Transportation Science 39 (1), 104-118.

Bräysy, O., Gendreau, M., 2005b. Vehicle routing problem with time windows, part ii: Metaheuristics. Transportation Science 39 (1), 119-139. 
Brizzolara, D., Toth, A., 2016. The emergence of truck platooning. In: Baltic Transport Journal, 3rd Edition.

Campbell, J. F., 1990. Freight consolidation and routing with transportation economies of scale. Transportation Research Part B: Methodological 24 (5), 345-361.

Chao, I.-M., 2002. A tabu search method for the truck and trailer routing problem. Computers I\& Operations Research.

Chardaire, P., McKeown, G., Verity-Harrison, A., Richardson, S., 2005. Solving a time-space network formulation for the convoy movement problem. Operations Research 53 (2), 219-230.

Chen, M., Hu, Q., Mackin, C., Fisac, J. F., Tomlin, C. J., 2015. Safe platooning of unmanned aerial vehicles via reachability. In: Decision and Control (CDC), 2015 IEEE 54th Annual Conference on. IEEE, pp. 4695-4701.

Chen, W., Mes, M., Schutten, M., Quint, J., 2016. A ride sharing problem with meeting points and return restrictions. Beta Publication series.

Chen, Z., He, F., Yin, Y., Du, Y., 2017. Optimal design of autonomous vehicle zones in transportation zones. Transportation Research Part B 99, 44-61.

Cheng, S.-F., Nguyen, D. T., Lau, H. C., 2012. A mechanism for organizing last mile service using non dedicated fleet. WI-IAT 2012: IEEE/WIC/ACM International Conference on Intelligent Agent Technology, 4-7 December 2012, Macau: Proceedings., 85-89.

Cordeau, J.-F., Gendreau, M., Laporte, G., 1997. A tabu search heuristic for periodic and multi-depot vehicle routing problems. Networks 30 (2), 105-119.

Cordeau, J.-F., Gendreau, M., Laporte, G., Potvin, J.-Y., Semet, F., 2002. A guide to vehicle routing heuristics. The Journal of the Operations Research Society 53 (5), $512-522$.

Cordeau, J.-F., Laporte, G., 2007. The dial-a-ride problem: models and algorithms. Ann Oper Res 153, 29-46. 
Crainic, T. G., 2000. Service network design in freight transportation. European Journal of Operational Research 122, 272-288.

Crainic, T. G., Kim, K. H., 2007. Intermodal transportation. Handbooks in operations research and management science $14,467-537$.

Crevier, B., Cordeau, J. F., Laporte, G., 2007. The multi-depot vehicle routing problem with inter-depot routes. European Journal of Operational Research 176 (2), 756-773.

Croxton, K. L., Gendron, B., Magnanti, T. L., 2003. Models and methods for merge-in-transit operations. Transportation Science 37 (1), 1-22.

Cruijssen, F., Dullaert, W., Fleuren, H., 2007. Horizontal cooperation in transport and logistics a literature review. Transportation Journal 46 (3), 22-39.

Daduna, J. R., Voß, S., 1995. Practical experiences in schedule synchronization. Computer-Aided Transit Scheduling, 39-55.

Dall'Orto, L. C., Crainic, T. G., Leal, J. E., Powell, W. B., 2006. The single-node dynamic service scheduling and dispatching problem. European journal of operational research $170(1), 1-23$.

Derigs, U., Pullman, M., Vogel, U., 2013. Truck and trailer routing - problems, heuristics and computational experience. Computers and Operations Research 40, 536-546.

Domschke, W., 1989. Schedule synchronization for public transit networks. Operations-Research-Spektrum 11, 17-24.

Drexl, M., 2011. Applications of the vehicle routing problem with trailers and transshipments. Tech. rep., Johannes Gutenberg University Mainz and Fraunhofer Centre for Applied Research on Supply Chain Services SCS.

Drexl, M., 2012. Synchronization in vehicle routing - a survey of VRPs with multiple synchronization constraints. Transportation Science 46 (3), 297-316. 
Dumas, Y., Soumis, F., Desroiers, J., 1990. Technical note - optimizing the schedule for a fixed vehicle path with convex inconvenience costs. Transportation Science 24 (2), $145-152$.

Furuhata, M., Dessouky, M., Ordez, F., Brunet, M.-E., Wang, X., Koenig, S., 2013.

Ridesharing: The state-of-the-art and future directions. Transportation Research Part B $57,28-46$.

Eckhardt, J., Aarts, L., van Vliet, A., Alkim, T., 2016. European truck platooning challenge 2016, lessons learnt.

Elkind, E., Rothe, J., 2016. Economics and computation. Springer.

Ernst, A. T., Jiang, H., Krishnamoorthy, M., Sier, D., 2004. Staff scheduling and rostering: A review of applications, methods and models. European Journal of Operations Research $153(1), 3-27$.

European Commission, 2016. Climate action.

URL http://ec.europa.eu/clima/policies/transport/vehicles/index_en.htm

Fagin, R., Williams, J. H., 1983. A fair carpool scheduling algorithm. IBM Journal of Research and Development 27 (2), 132-140.

Farokhi, F., Johansson, K. H., 2013. A game-theoretic framework for studying truck platooning incentives. In: Proceedings of the 16th International IEEE Annual Conference on Intelligent Transportation Systems (ITSC 2013), The Hague, The Netherlands. pp. 1253-1260.

Farokhi, F., Johansson, K. H., 2014. Investigating the interaction between traffic flow and vehicle platooning using a congestion game. In: Proceedings of the 19th World Congress The International Federation of Automatic Control Cape Town, South Africa. pp. $4170-4177$.

Frisk, M., Göthe-Lundgren, M., Jörnsten, K., Rönnqvist, M., 2010. Cost allocation in collaborative forest transportation. European Journal of Operational Research 205 (2), $448-458$. 
Gansterer, M., Hartl, R. F., 2017. Collaborative vehicle routing: a survey. arXiv preprint arXiv:1706.05254v1.

Gendreau, M., Laporte, G., Séguin, R., 1996. Stochastic vehicle routing. European Journal of Operational Research 88, 3-12.

Glaeser, E. L., 2007. Introduction. In: Glaeser, E. L. (Ed.), Agglomeration Economics. University of Chicago Press, Ch. 1, pp. 1-14.

Goel, A., 2010. Truck driver scheduling in the European Union. Transportation Science $44(4), 429-441$.

Goel, A., 2014. Hours of service regulation in the United States and the 2013 rule change. Transport Policy 33, 48-55.

Goel, A., Archetti, C., Savelsbergh, M., 2012. Truck driver scheduling in Australia. Computers \& Operations Research 39, 1122-1132.

Goel, A., Rousseau, L.-M., 2012. Truck driver scheduling in Canada. Journal of Scheduling $15(6), 783-789$.

Government-UK, 2016. Driving hours - EU rules. URL https://www.gov.uk/drivers-hours/eu-rules

Guajardo, M., Rönnqvist, M., 2016. A review on cost allocation methods in collaborative transportation. International transactions in operational research 23, 371-392.

Gupta, M., Judge, P., Ammar, M., 2003. A reputation system for peer-to-peer networks. In: Proceedings of the 13th international workshop on Network and operating systems support for digital audio and video. ACM, pp. 144-152.

Hall, R. W., 1987. Consolidation strategy: Inventory, vehicles and terminals. Journal of Business Logistics 8 (2), 57-73.

Heeswijk, W. V., Mes, M., Schutten, J., Zijm, W., 2016. Freight consolidation in intermodal networks with reloads. Flexible Services and Manufacturing Journal, 1-34. 
Heikoop, D., de Winter, J. C. F., van Arem, B., Stanton, N. A., 2017. Effects of platooning on signal-detection performance, workload, and stress: A driving simulator study. Applied Egronomics 60, 116-127.

Hjamdähl, M., Krupenia, S., Thorslund, B., 2017. Driver behaviour and driver experience of partial and fully automated truck platooning a simulator study. European Transport Research Review 9 (8).

Hollis, B. L., Forbes, M. A., Douglas, B. E., 2006. Vehicle routing and crew scheduling for metropolitan mail distribution at australia post. European Journal of Operational Research 173 (1), 133-150.

Huber, S., Geiger, M. J., 2014. Swap body vehicle routing problem: A heuristic solution approach. In: International Conference on Computational Logistics. Springer, pp. 16-30.

Iliopoulos, C. S., Rahman, M. S., 2008. Algorithms for computing variants of the longest common subsequence problem. Theoretical Computer Science 395, 255-267.

Janssen, R., Zwijnenberg, H., Blankers, I., de Kruijff, J., 2015. Truck platooning: Driving the future of transportation. Tech. rep., TNO.

Kavathekar, P., Chen, Y., 2011. Vehicle platooning: A brief survey and categorization. In: ASME 2011 International Design Engineering Technical Conferences and Computers and Information in Engineering Conference. American Society of Mechanical Engineers, pp. 829-845.

Kenyon, A. S., Morton, D. P., 2003. Stochastic vehicle routing with random travel times. Transportation Science 37 (1), 69-82.

Kilcarr, S., 2016. Driverless trucks: Where they'll work, where they won't.

URL http://m.fleetowner.com/technology/ driverless-trucks-where-they-ll-work-where-they-won-t

Kim, K. H., Bae, J. W., 2004. A look-ahead dispatching method for automated guided vehicles in automated port container terminals. Transportation Science 38 (2), 224-234. 
Krajewska, M. A., Kopfer, H., Laporte, G., Ropke, S., Zaccour, G., 2008. Horizontal cooperation among freight carriers: request allocation and profit sharing. Journal of the Operations Research Society 59 (11), 1483-1491.

Kumar, P. R., Narendran, T., 2010. Convoy movement probleman optimization perspective. Innovations in Defence Support Systems1, 79-93.

Lammert, M., Duran, A., Dies, J., Burton, K., Nicholson, A., 2014. Effect of platooning on fuel consumption of class 8 vehicles over a range of speeds, following distances, and mass. SAE Int. J. Commer. Veh. 7 (2), 626-639.

Laporte, G., 1992. The vehicle routing problem: An overview of exact and approximate algorithms. European Journal of Operational Research 59, 345-358.

Laporte, G., Gendreau, M., Potvin, J.-Y., Semet, F., 2000. Classical and modern heuristics for the vehicle routing problem. International transactions in operational research $7(4-5), 285-300$.

Laporte, G., Louveaux, F., Mercure, H., 1992. The vehicle routing problem with stochastic travel times. Transportation Science 26 (3), 161-170.

Larburu, M., Sanchez, J., Rodriguez, D. J., 2010. Safe road trains for environment: Human factors aspects in dual mode transport systems. In: ITS World Congress, Busan, Korea.

Larson, J., Kramer, C., Lang, K.-Y., Johannson, K. H., 2013. Coordinated route optimization for heavy duty vehicle platoons. In: Proceedings of the 16th International IEEE Annual Conference on Intelligent Transportation Systems (ITSC 2013), The Hague, The Netherlands, October 6-9, 2013. pp. 1196-1202.

Larson, J., Munson, T., Sokolov, V., 2016. Coordinated platoon routing in a metropolitan network.

URL http://www.mcs .anl.gov/papers/P6010-0516.pdf

Larsson, E., Sennton, G., Larson, J., 2015. The vehicle platooning problem: Computation complexity and heuristics. Transportation Research Part C 60, 258-277. 
Lauf, O., 2017. NMT cördineert nieuw maritiem europees innovatieproject 'NOVIMAR'. URL http://maritimetechnology.nl/ nmt-coordineert-nieuw-maritiem-europees-innovatieproject-novimar/

Levofsky, A., Greenberg, A., 2001. Organized dynamic ride sharing: the potential environmental benefits and the opportunity for advancing the concept. Transportation Research Board 2001 Annual Meeting, 7-11.

Liang, K.-Y., Mårtensson, J., Johansson, K. H., 2013. When is it fuel efficient for a heavy duty vehicle to catch up with a platoon? In: 7th IFAC Symposium on Advances in Automotive Control The International Federation of Automatic Control September 4-7, 2013. Tokyo, Japan. pp. 738-743.

Liang, K.-Y., Mårtensson, J., Johansson, K. H., 2014. Fuel-saving potentials of platooning evaluated through sparse heavy-duty vehicle position data. In: 2014 IEEE Intelligent Vehicles Symposium (IV) June 8-11, 2014. Dearborn, Michigan, USA. pp. 1061-1068.

Liang, K.-Y., Mårtensson, J., Johansson, K. H., 2016. Heavy-duty vehicle platoon formation for fuel efficiency. IEEE Transactions on Intelligent Transportation Systems 17 (4), 1051-1061.

Lim, A., Wang, F., 2005. Multi-depot vehicle routing problem: A one-stage approach. IEEE transactions on Automation Science and Engineering 2 (4), 397-402.

Lioris, J., Pedarsani, R., Tascikaraoglu, F. Y., Varaiya, P., 2017. Platoons of connected vehicles can double throughput in urban roads. Transportation Research Part C 77, $292-305$.

Lozano, S., Moreno, P., Adenso-Díaz, B., Algaba, E., 2013. Cooperative game theory approach to allocating benefits of horizontal cooperation. European Journal of Operational Research 229 (2), 444-452.

Lum, O., Chen, P., Wang, X., Golden, B., Wasil, E., 2015. A heuristic approach for the swap-body vehicle routing problem. In: 14th INFORMS Computing Society Conference Richmond, Virginia, January 11-13, 2015. pp. 172-187. 
Meisel, F., Kopfer, H., 2014. Synchronized routing of active and passive means of transport. OR Spectrum 36, 297-322.

Meisen, P., Seidl, T., Henning, K., 2008. A data mining technique for the planning and organization of truck platoons. International Conference on Heavy Vehicles (HVParis 2008), Heavy Vehicle Transport Technology. ISTE I\& Wiley.

Merchant, D. K., Nemhauser, G. L., 1978. A model and an algorithm for the dynamic traffic assignment problems. Transportation Science 12 (3), 183-199.

Min, H., Cooper, M., 1990. A comparative review of analytical studies on freight consolidation and backhauling. Logistics and Transportation Review 26 (2), 149-169.

Minett, P., 2013. Flexible carpooling to transit stations. Tech. rep., Transportation research board of the national academies.

Ministry of Transport - Singapore, 2017. Singapore to start truck platooning trials. URL https://www.mot.gov.sg/News-Centre/News/2017/ Singapore-to-start-truck-platooning-trials/

Miranda-Bront, José, J., Curcio, B., Méndez-Díaz, I., Montero, A., Pousa, F., Zabala, P., 2015. A cluster-first route-second approach for the swap body vehicle routing problem. Annals of Operations Research, 1-22.

Mui, L., Mohtashemi, M., Halberstadt, A., 2002. Notions of reputation in multi-agents systems: a review. In: Proceedings of the first international joint conference on Autonomous agents and multiagent systems: part 1. ACM, pp. 280-287.

Mukai, N., Watanabe, T., 2005. Dynamic transport services using flexible positioning of bus stations. In: Proceedings Autonomous Decentralized Systems 2002 ISADS 2005. IEEE, pp. 259-266.

Nobel Prize Committee, 2007. Mechanism design theory. URL http://www .nobelprize.org/nobel_prizes/economic-sciences/laureates/ 2007/advanced-economicsciences2007.pdf 
Nourmohammadzadeh, A., Hartmann, S., 2016. The fuel-efficient platooning of heavy duty

Raff, S., 1983. Routing and scheduling of vehicles and crews. Computers \& Operations Research 10 (2), 117-147. vehicles by mathematical programming and genetic algorithm. In: Martín-Vide, C., Mizuki, T., Vega-Rodríguez, M. (Eds.), Theory and Practice of Natural Computing. TPNC 2016. Lecture Notes in Computer Science. Vol. 10071. Springer.

Nowakowski, C., Schladover, S. E., Lun, X.-Y., Thompson, D., Kailas, A., 2015. Cooperative adaptive cruise control (cacc) for truck platooning: Operational concept alternatives. Tech. rep., California Partners for Advanced Transportation Technology UC Berkeley.

Parks, D., 2001. Iterative combinatorial auctions: Achieving economic and computational efficiency. Ph.D. thesis, University of Pennsylvania.

Patten, J., McAucliffe, B., Mayda, W., Tanguay, B., 2012. Review of aerodynamic drag reduction devices for heavy trucks and buses. Tech. rep., National Research Council Canada.

Peloton Technology, 2016. Industry report: Platooning systems such as from peloton offer significant fuel efficiency i\& safety gains.

URL http://peloton-tech.com/nacfe_release

Pillac, V., Gendreau, M., Guéret, C., Medaglia, A. L., 2013. A review of dynamic vehicle routing problems. European Journal of Operational Research 225 (1), 1-11.

Powell, W. B., 1996. A stochastic formulation of the dynamic assignment problem, with an application to truckload motor carriers. Transportation Science 30 (3), 195-219.

Prins, C., Lacomme, P., Prodhon, C., 2014. Order-first split-second methods for vehicle routing problems: A review. Transportation Research Part C: Emerging Technologies 40, 179-200. 
Renaud, J., Laporte, G., Boctor, F. F., 1996. A tabu search heuristic for the multi-depot vehicle routing problem. Computers \& Operations Research 23 (3), 229-235.

Resnick, P., Zeckhauser, R., 2015. Trust among strangers in internet transactions: Empirical analysis of ebay's reputation system. The Economics of the Internet and E-commerce, 127-157.

Reyes, D., Savelsbergh, M., Toriello, A., 2016. Vehicle routing with roaming delivery locations. Optimization Online, 1-27.

Richert, D., Cortés, J., 2012. Optimal leader allocation in UAV formation pairs under no-cost switching. In: American Control Conference (ACC), 2012. IEEE, pp. 3297-3302.

Roland Berger, 2016. Automated trucks - the next big disruptor in the automotive industry. Tech. rep., Roland Berger.

Roodbergen, K. J., Vis, I. F., 2009. A survey of literature on automated storage and retrieval systems. European journal of operational research 194 (2), 343-362.

SAE International, 2016. Taxonomy and definitions for terms related to on-road motor vehicle automated driving systems.

Salazar-Aguilar, M. A., Langevin, A., Laporte, G., 2011. Synchronized arc routing for snow plowing operations. Computers \& Operations Research 39, 1432-1440.

Savelsbergh, M., Sol, M., 1995. The general pick-up and delivery problem. Transportation Science 29 (1), 17-29.

Schladover, S. E., Nowakowski, C., Lu, X.-Y., Felis, R., 2015. Cooperative adaptive cruise control (CACC) definitions and operating concepts. Proceedings of the 94th Annual TRB Meeting. Transportation Research Board.

Schwartz, A., Scott, R. E., 2003. Contract theory and the limits of contract law. The Yale law journal 113 (3), 541-619. 
Scora, G., Barth, M., 2006. Comprehensive modal emissions model (CMEM), version 3.01. User guide. Centre for Environmental Research and Technology. University of California, Riverside.

Segata, M., Bloessl, B., Joerer, S., Dressler, F., Cigno, R. L., 2014. Supporting platooning maneuvers through ivc: An initial protocol analysis for the JOIN maneuver. In: 2014 11th Annual Conference on Wireless On-demand Network Systems and Services (WONS). IEEE, pp. 130-137.

Sokolov, V., Larson, J., Munson, T., Auld, J., Karbowski, D., 2017. Platoon formation maximization through centralized routing and departure time coordination. arXiv preprint arXiv:1701.01391.

Stiglic, M., Agatz, N., Savelsbergh, M., Gradisar, M., 2015. The benefits of meeting points in ride sharing systems. Transportation Research Part B: Methdological 82, 36-53.

TNO, 2016. Truck platooning vision 2025.

URL https://www . eutruckplatooning.com/Workspace/Conference\%2BTruck\% 2BPlatooning\%2BChallenge\%2B7\%2BApril\%2B2016/handlerdownloadfiles . ashx\% $3 F i d n v \% 3 D 499128+\& c d=1 \& h l=e n \& c t=c l n k \& g l=n l$

Tsugawa, S., 2014. Results and issues of an automated truck platoon within the energy its project. Intelligent Vehicles Symposium Proceedings, 2014 IEEE, 642-647.

TU Delft 3mE, 2017. Major investment in platooning with autonomous cargo ships. URL http://www.3me.tudelft.nl/en/current/latest-news/article/detail/ forse-investering-voor-platooning-met-autonoom-varende-vrachtschepen/

Tuson, A., Harrison, S., 2005. Problem difficulty of real instances of convoy planning. Journal of the Operational Research Society 56, 763-775.

UNSW Engineering, 2016. Western Australia to host truck platooning trial. URL http://www.snap.unsw.edu.au/news/ western-australia-to-host-truck-platooning-trial 
Valdés, F., Iglesias, R., Espinosa, F., Rodrguez, M. A., 2011. An efficient algorithm for

Xu, P. J., Allgor, R., Graves, S. C., 2009. Benefits of reevaluating real-time order fulfillment decisions. Manufacturing and Service Operations Management 11 (2), 340-355.

Xu, X., 2013. Collaboration mechanism in the horizontal logistics collaboration. Ph.D. thesis, Ecole Nationale Superieure des Mines de Paris. 
Yamabe, S., Zheng, R., Nakano, K., Suda, Y., Takagi, T., Kawahara, S., 2012. Analysis on behaviors of a driver in the system failure in forming automatic platooning of trucks from manual driving. In: 19th ITS World Congress.

Yamada, T., Russ, B. F., Castro, J., Taniguchi, E., 2009. Designing multimodal freight transportation networks: A heuristic approach and applications. Transportation Science $43(2), 129-143$.

Zabat, M., Stabile, N., Frascaroli, S., Browand, F., 1995. The aerodynamic performance of platoons: Final report. Tech. rep., California PATH project, University of California Berkeley.

Zhang, W., Jenelius, E., Ma, X., 2017. Freight transport platoon coordination and departure time scheduling under travel time uncertainty. Transportation Research Part E $98,1-12$. 